\title{
Inferences on the solar envelope with high-degree modes
}

\author{
M. P. Di Mauro ${ }^{1,2}$, J. Christensen-Dalsgaard ${ }^{2,3}$, M. C. Rabello-Soares ${ }^{4}$, and S. Basu ${ }^{5}$ \\ 1 Osservatorio Astrofisico di Catania, Via S. Sofia 78, 95123 Catania, Italy \\ 2 Teoretisk Astrofysik Center, Danmarks Grundforskningsfond, 8000 Aarhus C, Denmark \\ 3 Institut for Fysik og Astronomi, Aarhus Universitet, Ny Munkegade bygn. 520, 8000 Aarhus C, Denmark \\ e-mail: jcd@ifa.au.dk \\ 4 Hansen Experimental Physics Laboratory, Stanford University, Stanford, CA 94305, USA \\ e-mail: csoares@tucano.Stanford.EDU \\ 5 Astronomy Department, Yale University, New Haven CT 06520-8101, USA \\ e-mail: basu@astro.yale.edu
}

Received 27 November 2001 / Accepted 4 January 2002

\begin{abstract}
We investigate the structure of the Sun by helioseismic inversion of a set of p-mode frequencies which includes new precise observations of modes with high degree $(l<1000)$ obtained from the MDI instrument on the SOHO satellite (Rhodes et al. 1998). Such data have the potential to improve the resolution of the solar structure in the near-surface region, to provide detailed tests of the equation of state and constrain the envelope helium abundance. In order to suppress the uncertainties in the treatment of the surface layers in helioseismic inversion procedures, we introduce here the use of a new surface term, developed on the basis of higher-order asymptotic theory of acoustic modes and suitable for the handling of high-degree mode frequencies.
\end{abstract}

Key words. Sun: helioseismology - Sun: interior

\section{Introduction}

Helioseismic inversions of observed acoustic frequencies, available from a variety of helioseismology experiments on Earth and in space, have proved to be an extremely powerful tool for the investigation of the internal structure and dynamics of the Sun. Inversion results have, so far, confirmed that the solar structure is remarkably close to the predictions of the standard solar model, but have also suggested further refinements.

The detailed structure of the convection zone and of the near-surface region is still quite uncertain, since there remains substantial ambiguity associated with modelling the convective flux, explaining the excitation and damping of the solar oscillations, defining an appropriate equation of state to describe the thermodynamic properties of the solar structure, as well as in the treatment of nonadiabatic effects on the oscillations. In most cases, in fact, the theoretical frequencies are calculated in the adiabatic approximation, which is certainly inadequate in the nearsurface region, where the thermal time scale becomes comparable with the oscillation period.

Send offprint requests to: M. P. Di Mauro,

e-mail: mdm@ct.astro.it
Recently, Rabello-Soares et al. (2000) have demonstrated that the solar envelope can be probed with sufficiently high spatial resolution through inversion of highdegree acoustic modes $(l \geq 100)$, trapped near the solar surface. Unfortunately, there is no currently accepted procedure in helioseismic inversions to treat high-degree modes, which appear strongly affected by uncertainties associated with the treatment of surface layers.

In the absence of any acceptable theory to describe the physics of the layers near the solar photosphere, for helioseismic inversions it is usually assumed that uncertainties will give rise to frequency shifts. In order to compensate for these frequency shifts, an unknown function $F_{\text {surf }}$, the so-called surface term, is usually added to the equation governing helioseismic inversions (Dziembowski et al. 1990), such that

$\left(\frac{\delta \omega_{n, l}}{\omega_{n, l}}\right)_{\mathrm{surf}}=\frac{F_{\mathrm{surf}}}{Q_{n, l}}$

where the $\left(\delta \omega_{n, l} / \omega_{n, l}\right)_{\text {surf }}$ are the relative differences between the frequencies of the Sun and the reference model due only to errors in modelling the surface layers, the indices $n$ and $l$ specifying respectively the radial order and the harmonic degree of the mode. The term $Q_{n, l}$ is the 
inertia of the modes, normalized by the inertia of a radial mode of the same frequency. The function $F_{\text {surf }}$ must be determined as part of the analysis of the frequency differences. It should resemble, in practice, the differences $Q_{n, l}\left(\delta \omega_{n, l} / \omega_{n, l}\right)_{\text {surf }}$.

The appropriate mathematical expression for the $F_{\text {surf }}$ function is traditionally obtained by considering the asymptotic theory of solar p-modes (e.g. ChristensenDalsgaard et al. 1989), according to which the relative frequency differences between two models, or between the Sun and a model, can be obtained as the sum of two functions which depend respectively on the frequency and on $\omega / L$ :

$S_{n, l} \frac{\delta \omega_{n, l}}{\omega_{n, l}} \simeq H_{1}\left(\frac{\omega_{n, l}}{L}\right)+H_{2}\left(\omega_{n, l}\right)$,

where $L=l+1 / 2$ and $S_{n, l}$ is a scaling factor closely related to $Q_{n, l}$. Christensen-Dalsgaard et al. (1989) pointed out that $H_{1}\left(\omega_{n, l} / L\right)$ and $H_{2}\left(\omega_{n, l}\right)$ can be obtained separately, by means of a fit to the scaled p-mode frequency differences. In Eq. (2) the term in $H_{1}$ is determined by the difference in sound speed in the bulk of the star, while the second term reflects differences in the near-surface layers. In this asymptotic limit, therefore,

$S_{n, l}\left(\frac{\delta \omega_{n, l}}{\omega_{n, l}}\right)_{\text {surf }} \simeq H_{2}\left(\omega_{n, l}\right)$

thus the surface term becomes a function of the frequency alone, $F_{\text {surf }} \simeq f(\omega)$. More generally, this behaviour follows physically from the fact that low- and moderate-degree modes propagate nearly vertically near the surface; thus, their behaviour in this region is essentially independent of degree and depends only on frequency.

The expression (2) is certainly valid to a first approximation, at least when the degree $l$ is not too large. However, the accuracy of current measurements of frequencies requires the inclusion of higher-order terms in the asymptotic treatment, and in particular the dependence on the degree should be taken explicitly into account when high-degree modes are considered (Antia 1995).

In this paper we adopt a surface term obtained through a second-order asymptotic approximation developed by Brodsky \& Vorontsov (1993) for adiabatic nonradial p-modes of a spherically symmetric star. This approach not only results in a large improvement of the fit between observed and theoretical frequencies, but also has an effect on the helioseismic inversions and on the determination of the properties of the Sun's interior.

\section{The frequency differences and the surface term}

Analysis of the oscillation equations (e.g. ChristensenDalsgaard \& Thompson 1997) shows that near-surface model differences cause frequency differences that are very small at low frequency and generally increase in magnitude with increasing frequency. The actual frequency

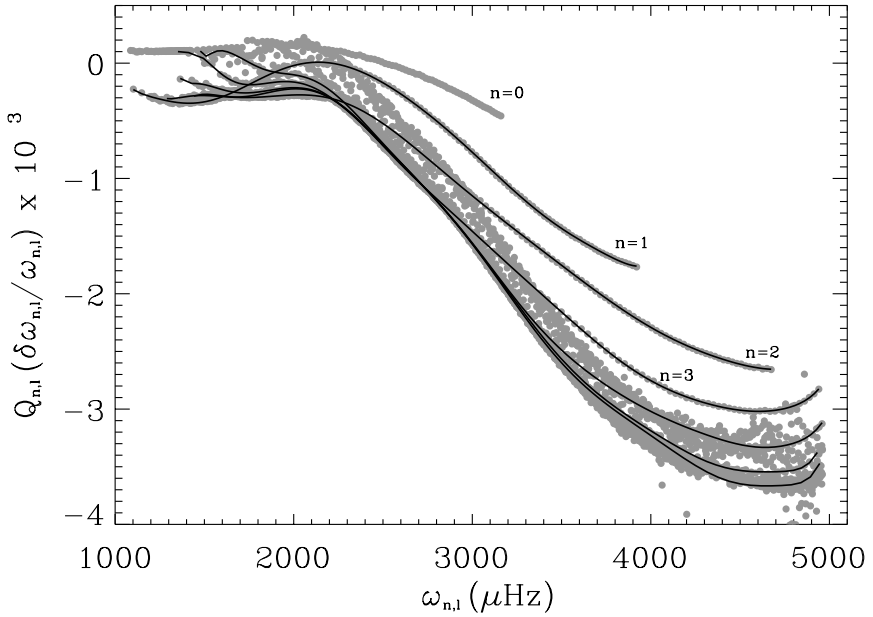

Fig. 1. Relative differences between the observed frequencies of Rhodes et al. (1998) and theoretical frequencies for Model S of Christensen-Dalsgaard et al. (1996), scaled by the normalized mode inertia $Q_{n, l}$ and plotted as a function of frequency (grey dots); the differences are shown in the sense (Sun) - (model). The black solid lines represent the fits of the expression (6) to the p-mode frequency differences using $\Lambda=8$; note that this expression is not relevant for the f-modes, with $n=0$.

differences, scaled by $Q_{n, l}$, between the Sun and a solar model that are shown in Fig. 1 share this property. The frequency dependence results in part from uncertainties in the mode physics, but also from the real differences between the structure of the Sun and the reference model. The $l$-dependence of the frequency differences is mainly associated with the variation of the mode inertia, since modes with higher $l$ penetrate less deeply and hence have a smaller inertia. Thus, high-degree modes are affected more strongly by the near-surface uncertainties (e.g. Dziembowski et al. 1990; Antia 1995; Gough \& Vorontsov 1995). As shown in Fig. 1, this degree dependence is largely, but not completely, suppressed by scaling the frequency differences by $Q_{n, l}$. At intermediate and high degree there remains a variation with degree, reflected in the fact that the scaled frequency differences for modes of low order $n$ fall on distinct curves. We note that the frequencies of the f modes (with $n=0$ ) depend only weakly on the hydrostatic structure of the model (e.g. Gough 1993); thus the significant differences for these modes shown in Fig. 1 indicate that the computed frequencies are affected also by inadequacies in the mode physics.

A careful and detailed application of the JWKB asymptotic theory of stellar acoustic oscillation led Brodsky \& Vorontsov (1993) to formulate an accurate second-order asymptotic expression for the eigenfrequencies of solar acoustical oscillations:

$\int_{r_{\mathrm{t}}}^{R_{\odot}}\left(1-\frac{L^{2} c^{2}}{r^{2} \omega^{2}}\right)^{1 / 2} \mathrm{~d} r+\frac{1}{\omega^{2}} \Phi(\tilde{w}) \simeq \pi \frac{n+\alpha(\omega, \tilde{w})}{\omega}$,

where $\tilde{w}=L / \omega$ and $r_{\mathrm{t}}$, the lower limit of integration, is the lower turning point of the mode. The function 
$\Phi(\tilde{w})$ represents the term arising from the effects of buoyancy and the perturbation to the gravitational potential, although both of them appear to be small for modes that are confined to the convection zone. The surface phase shift $\alpha(\omega, \tilde{w})$ is approximately a function of frequency alone for low- and intermediate-degree modes, while for higher-degree modes the dependence of the phase shift on the degree $l$ becomes significant. Here we express the phase shift, as did Brodsky \& Vorontsov (1993), by an expansion in powers of $\tilde{w}^{2}$, which we have chosen to truncate at the third term (see also Gough \& Vorontsov 1995). Thus $\alpha(\omega, \tilde{w}) \simeq \alpha_{0}(\omega)+\tilde{w}^{2} \alpha_{2}(\omega)+\tilde{w}^{4} \alpha_{4}(\omega)$, where $\alpha(\omega, \tilde{w}) \simeq \alpha_{0}(\omega)$ is valid when low- and intermediatedegree modes are considered.

By considering the effect of small changes to the equilibrium structure in Eq. (4), one obtains that the frequency differences may be written as

$$
\begin{aligned}
S_{n, l} \frac{\delta \omega_{n, l}}{\omega_{n, l}} \simeq & H_{1}\left(\frac{\omega_{n, l}}{L}\right)+H_{2}\left(\omega_{n, l}\right) \\
& +H_{3}\left(\omega_{n, l}\right) \tilde{w}^{2}+H_{4}\left(\omega_{n, l}\right) \tilde{w}^{4} .
\end{aligned}
$$

Thus the relative frequency differences are given by the contributions of four different functions. Of these the first, as in Eq. (2), contains information about the sound speed in the solar interior, whereas the remaining three result from expanding the surface phase $\alpha(\omega, \tilde{w})$ and hence contain the contribution from the effects of the near-surface layers. The functions $H_{2}\left(\omega_{n, l}\right), H_{3}\left(\omega_{n, l}\right)$ and $H_{4}\left(\omega_{n, l}\right)$ can be expressed as expansions in terms of Legendre polynomials of the frequency $\omega$ while $H_{1}\left(\omega_{n, l} / L\right)$ may be approximated by a polynomial function of $\omega / L$. The coefficients of the expansions can be obtained by means of a non-linear least-squares fit of a sum of four linear combinations of Legendre polynomials to the scaled p-mode frequency differences.

We have carried out such fits, regarding the maximum value of the polynomial degree $\Lambda$ used in the expansions as a free parameter, assumed to be the same for all four terms. In practice, a suitable value of $\Lambda$ must be determined for any given data set. In the resulting fitting function the terms in $H_{2}-H_{4}$ can, by definition (1), be identified with the surface term $F_{\text {surf }}=f(\omega, \omega / L)$; this is then the new surface term which we use in the helioseismic inversion to account for uncertainties arising from the treatment of surface layers:

$$
\begin{aligned}
F_{\text {surf }} \simeq & \sum_{\lambda=1}^{\Lambda} b_{\lambda} \mathcal{P}_{\lambda}(\omega) \\
& +\sum_{\lambda=1}^{\Lambda} c_{\lambda} \mathcal{P}_{\lambda}(\omega) \tilde{w}^{2}+\sum_{\lambda=1}^{\Lambda} d_{\lambda} \mathcal{P}_{\lambda}(\omega) \tilde{w}^{4} .
\end{aligned}
$$

Here the $\mathcal{P}_{\lambda}$ are essentially Legendre polynomials of degree $\lambda$, with argument scaled to be in the interval $[-1,1]$ for the modes considered.

In the current analyses the fitting has been carried out with different maximum degrees $\Lambda$, which was increased until the residuals $r_{n, l}=Q_{n, l} \delta \omega_{n, l} / \omega_{n, l}-F_{\text {surf }}$ could no

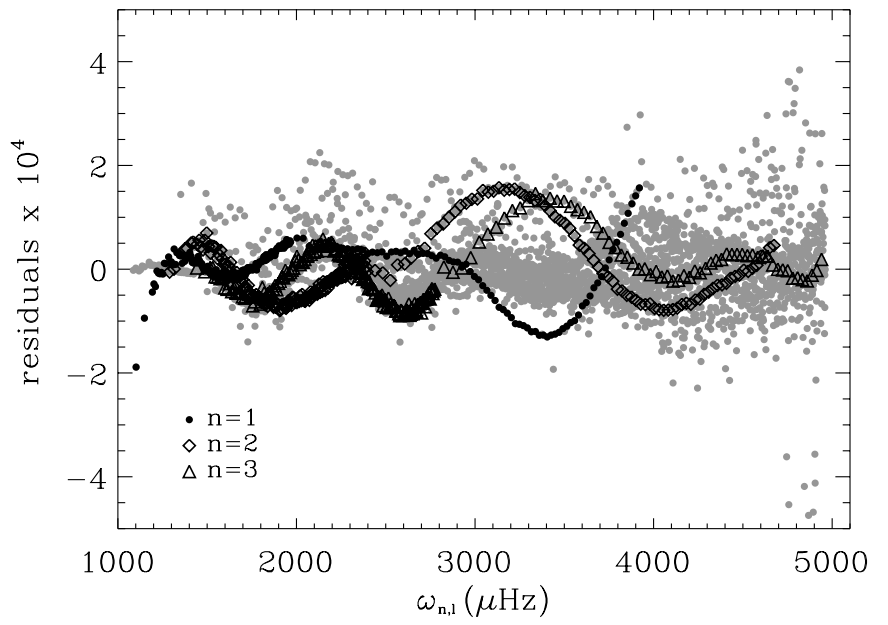

Fig. 2. The residuals $r_{n, l}=Q_{n, l} \delta \omega_{n, l} / \omega_{n, l}-F_{\text {surf }}$ plotted as function of frequency, as obtained by the fits shown in Fig. 1. Here the maximum polynomial degree used is $\Lambda=8$. Only residuals for acoustic modes (with $n \geq 1$ ) are shown.

longer be improved significantly. We found that fits with $\Lambda=8$ seem to provide an adequate representation of the scaled frequency differences in Fig. 1, leaving small residuals (see Fig. 2) with a modest, but still significant dependence on radial order; use of higher values of $\Lambda$ tends to increase the residuals.

\section{Helioseismic inversions for solar structure}

\subsection{Sound speed and density}

An inversion for solar structure is usually based on the linearization of the equations of stellar oscillations around a known reference model, under the assumption of hydrostatic equilibrium. This results in integral equations that can be used in an inverse procedure to determine the corrections which have to be imposed on the reference model in order to obtain the observed oscillation frequencies. Obviously, the results of the inversion depend both on the mode selection $i \equiv(n, l)$ and on the observational errors which characterize the mode set $(i=1, \ldots, M)$ to be inverted.

The relative differences $\delta \omega_{i} / \omega_{i}$ between the frequencies of the Sun and the model are related to the differences $\left(\delta c^{2} / c^{2}, \delta \rho / \rho\right)$ in sound speed $c$ and density $\rho$ between the structure of the Sun and the reference model through the following integral equation (e.g. Dziembowski et al. 1990):

$$
\begin{aligned}
\frac{\delta \omega_{i}}{\omega_{i}}= & \int_{0}^{R_{\odot}} K_{c^{2}, \rho}^{i}(r) \frac{\delta c^{2}}{c^{2}}(r) \mathrm{d} r \\
& +\int_{0}^{R_{\odot}} K_{\rho, c^{2}}^{i}(r) \frac{\delta \rho}{\rho}(r) \mathrm{d} r+\frac{F_{\text {surf }}}{Q_{i}}+\varepsilon_{i}
\end{aligned}
$$

where $r$ is the radial distance from the centre, $R_{\odot}$ is the solar photospheric radius and $\varepsilon_{i}$ the observational 
errors in the data, which are assumed to be independent and Gaussian-distributed with zero mean and variances $\sigma_{i}^{2}$. The kernels $K_{c^{2}, \rho}^{i}$ and $K_{\rho, c^{2}}^{i}$ are calculated, for each mode $i$, from the equilibrium model quantities and oscillation eigenfunctions.

Equation (7), which forms the basis for the linearized structure inversion problem, involves three unknown functions: $\delta c^{2} / c^{2}, \delta \rho / \rho$ and $F_{\text {surf }}$. However, the number of the unknown functions can be reduced to one by adopting the method of the optimally localized averages.

This method aims at solving Eq. (7) by estimating a localized weighted average of the unknown quantity, $\delta c^{2} / c^{2}$ or $\delta \rho / \rho$, at selected target radii $r_{0}$, by means of linear combinations of the data $\delta \omega_{i} / \omega_{i}$ with coefficients $\alpha_{i}\left(r_{0}\right)$, chosen such as to localize the solution while suppressing the contributions from the other terms. The solutions are characterized by the so-called averaging kernel and by the cross-term kernel, which in the case of the sound-speed inversion are expressed, respectively, by

$\mathcal{K}\left(r_{0}, r\right)=\sum_{i=1}^{M} \alpha_{i}\left(r_{0}\right) K_{c^{2}, \rho}^{i}(r)$

and

$\mathcal{C}\left(r_{0}, r\right)=\sum_{i=1}^{M} \alpha_{i}\left(r_{0}\right) K_{\rho, c^{2}}^{i}(r)$.

The cross-term kernel measures the influence of the contribution from $\delta \rho / \rho$ on the inferred $\delta c^{2} / c^{2}$. Analogous relations can be obtained for the density inversion, so that the averaging kernel is expressed as a linear combination of the mode kernels $K_{\rho, c^{2}}^{i}$, while the cross term, which represents the contribution of the sound speed to the inferred density difference, is given as a linear combination of $K_{c^{2}, \rho}^{i}$.

In particular, Eq. (7) has been solved using the Subtractive Optimally Localized Averages (SOLA) method (Pijpers \& Thompson 1992, 1994), fitting the averaging kernel to a target function $\mathcal{G}\left(r_{0}, r\right)$ of appropriate width and centered at the target radius; thus the inversion coefficients $\alpha_{i}\left(r_{0}\right)$ are chosen to minimize

$$
\begin{aligned}
\int_{0}^{R_{\odot}}\left[\mathcal{K}\left(r_{0}, r\right)-\mathcal{G}\left(r_{0}, r\right)\right]^{2} \mathrm{~d} r+ & \beta \int_{0}^{R_{\odot}} \mathcal{C}^{2}\left(r_{0}, r\right) g(r) \mathrm{d} r \\
& +\mu \sum_{i=1}^{M} \alpha_{i}^{2}\left(r_{0}\right) \sigma_{i}^{2}, \quad
\end{aligned}
$$

where $g(r)$ is a suitably increasing function of radius aimed at suppressing the surface structure in the crossterm kernel; we have used $g(r)=(1+r / R)^{4}$. The target functions were chosen to be Gaussians with widths proportional to the sound speed at $r=r_{0}$, to reflect the variation with depth in the wavelength of the eigenfunctions. Further details on the inversion method were provided by Rabello-Soares et al. (1999).

The coefficients $\alpha_{i}\left(r_{0}\right)$ should be chosen such as to suppress the contribution from the cross term, to localize the averaging kernel near $r=r_{0}$, to suppress the surface term, while limiting the error in the solution. This can be achieved by the use of the two parameters $\beta$, which controls the contribution of the cross-term kernel, and $\mu$, which is the trade-off parameter determining the balance between the demands of well-localized averaging kernels and a small error in the solution.

The contribution of $F_{\text {surf }}$ is suppressed by constraining the inversion coefficients to satisfy:

$$
\begin{aligned}
\sum_{i=1}^{M} \frac{\alpha_{i}\left(r_{0}\right)}{Q_{i}}\left[\mathcal{P}_{\lambda}\left(\omega_{i}\right)\right. & +\frac{c_{\lambda}}{b_{\lambda}} \mathcal{P}_{\lambda}\left(\omega_{i}\right) \tilde{w}_{i}{ }^{2} \\
+ & \left.+\frac{d_{\lambda}}{b_{\lambda}} \mathcal{P}\left(\omega_{i}\right) \tilde{w}_{i}{ }^{4}\right]=0, \quad \lambda=0,1, \ldots \Lambda,
\end{aligned}
$$

according to the definition of surface term given by the expression (6). Here the coefficients $b_{\lambda}, c_{\lambda}$ and $d_{\lambda}$ are obtained before the inversion by fitting the frequency differences to an expression of the form of Eq. (5).

We note that this implementation of the new surface term is not without problems: the constraints in Eq. (11) depend directly on the data through the ratios $c_{\lambda} / b_{\lambda}$ and $d_{\lambda} / b_{\lambda}$, and hence the properties of the inversion, such as errors and averaging kernels, have some dependence on the data values, unlike the usual inversion procedures. This problem could in principle be avoided by replacing the constraints in Eq. (11) by separate constraints based on each of the terms in Eq. (6):

$$
\begin{aligned}
& \sum_{i=1}^{M} \frac{\alpha_{i}\left(r_{0}\right)}{Q_{i}} \mathcal{P}_{\lambda}\left(\omega_{i}\right)=0 \\
& \sum_{i=1}^{M} \frac{\alpha_{i}\left(r_{0}\right)}{Q_{i}} \mathcal{P}_{\lambda}\left(\omega_{i}\right) \tilde{w}_{i}^{2}=0 \\
& \sum_{i=1}^{M} \frac{\alpha_{i}\left(r_{0}\right)}{Q_{i}} \mathcal{P}_{\lambda}\left(\omega_{i}\right) \tilde{w}_{i}^{4}=0, \quad \lambda=0,1, \ldots \Lambda
\end{aligned}
$$

We have not so far tested this implementation, however.

\subsection{Equation of state and helium abundance}

The equation of state can be investigated through the first adiabatic exponent $\Gamma_{1}$, the partial logarithmic derivative of pressure with respect to density at constant specific entropy. The solar plasma is almost an ideal gas, and the first adiabatic exponent is therefore close to $5 / 3$ in most of the interior. It deviates from this value in the zones of hydrogen and helium ionization, near the surface. Therefore, inversions of helioseismic data can be used, in particular, to study the equation of state and to probe the helium abundance in the solar envelope, as was demonstrated, e.g., by Gough (1984), Dziembowski et al. (1992), Kosovichev et al. (1992), and Basu \& Christensen-Dalsgaard (1997).

An integral equation analogous to Eq. (7) can be derived to determine the behaviour of $\left(\delta \Gamma_{1} / \Gamma_{1}\right)_{\text {int }}$, the relative intrinsic difference in $\Gamma_{1}$, at constant pressure $p$, density $\rho$ and composition, between the equation of state of 
the Sun and the one of the reference model, as did Basu \& Christensen-Dalsgaard (1997). The kernels for $\left(c^{2}, \rho\right)$ which appear in Eq. (7) can be converted to kernels for the set $\left(\Gamma_{1}, u, Y\right)$, where $u \equiv p / \rho$ and $Y$ is the helium abundance. After the conversion, Eq. (7) can be written as

$$
\begin{aligned}
\frac{\delta \omega_{i}}{\omega_{i}}= & \int_{0}^{R_{\odot}} K_{c^{2}, \rho}^{i}\left(\frac{\delta \Gamma_{1}}{\Gamma_{1}}\right)_{\mathrm{int}} \mathrm{d} r+\int_{0}^{R_{\odot}} K_{u, Y}^{i} \frac{\delta u}{u} \mathrm{~d} r \\
& +\int_{0}^{R_{\odot}} K_{Y, u}^{i} \delta Y \mathrm{~d} r+\frac{F_{\mathrm{surf}}}{Q_{i}}+\varepsilon_{i},
\end{aligned}
$$

where $\left(\delta \Gamma_{1} / \Gamma_{1}\right)_{\text {int }}$ is the deviation in $\Gamma_{1}$ that results from the differences in the equation of state alone, but not from the resulting change in solar structure. The term $\delta Y$ denotes the difference of the helium abundance in the convection zone between the Sun and the model.

It was noticed by Gough (1984) that the strong sensitivity of acoustic modes to the variation of the adiabatic exponent in the HeII ionization zone could allow also a seismic determination of the helium abundance in the outer layers of the Sun. Specifically, Eq. (13) may be inverted to determine $\delta Y$ in the helium ionization zones, following the procedure suggested by Dziembowski et al. (1990). Since the convection zone is fully mixed, this provides a measure of the value of the helium abundance in the solar envelope.

The adiabatic exponent and helium abundance differences have been inferred by the Multiplicative Optimally Localized Averages (MOLA) method (Backus \& Gilbert 1968), to allow a better localization of the solutions. Here the inversion coefficients are found by minimizing

$$
\begin{array}{r}
\int_{0}^{R} \mathcal{K}^{2}\left(r_{0}, r\right) J\left(r_{0}, r\right) \mathrm{d} r+\beta_{1} \int_{0}^{R_{\odot}} \mathcal{C}_{1}^{2}\left(r_{0}, r\right) g(r) \mathrm{d} r \\
+\beta_{2} \int_{0}^{R_{\odot}} \mathcal{C}_{2}^{2}\left(r_{0}, r\right) g(r) \mathrm{d} r+\mu \sum_{i=1}^{M} \alpha_{i}^{2}\left(r_{0}\right) \sigma_{i}^{2}
\end{array}
$$

where $\mathcal{K}^{2}\left(r_{0}, r\right)$ is the averaging kernel already introduced in Eq. (8), and

$$
\mathcal{C}_{1}\left(r_{0}, r\right)=\sum_{i=1}^{M} \alpha_{i}\left(r_{0}\right) K_{u, Y}^{i}(r),
$$

and

$\mathcal{C}_{2}\left(r_{0}, r\right)=\sum_{i=1}^{M} \alpha_{i}\left(r_{0}\right) K_{Y, u}^{i}(r)$,

are respectively the first and the second cross-term kernels. The parameters $\beta_{1}$ and $\beta_{2}$ control the contributions of $\delta u / u$ and $\delta Y$, respectively, and $\mu$ is a trade-off parameter which controls the effect of data noise. $J\left(r_{0}, r\right)$ is a weight function, which is zero for $r=r_{0}$ and large elsewhere, and $g(r)$ is again included to suppress surface structure in the first and second cross-term kernels.

The kernel $K_{Y, u}^{i}$ is given by

$$
K_{Y, u}^{i}=\left(\frac{\partial \ln \Gamma_{1}}{\partial Y}\right)_{p, \rho} K_{c^{2}, \rho}^{i} .
$$

It therefore follows that the inversion provides an inference of the combination

$$
\left(\frac{\delta \Gamma_{1}}{\Gamma_{1}}\right)_{\mathrm{int}}+\left(\frac{\partial \ln \Gamma_{1}}{\partial Y}\right)_{p, \rho} \delta Y
$$

and it appears not possible in general to separate the contribution of $\delta Y$ from $\left(\delta \Gamma_{1} / \Gamma_{1}\right)_{\text {int }}$. However, it is still possible to achieve some suppression of the effect of $\delta Y$, given that the magnitude of $\left(\partial \ln \Gamma_{1} / \partial Y\right)_{p, \rho}$, and hence $K_{Y, u}^{i}$, is negligible below $0.97 R_{\odot}$ (Rabello-Soares et al. 2000).

\section{Models and data}

Here we will show results obtained by using two reference models - Model $S_{\text {OPAL }}$ and Model $S_{\text {MHD }}$ - by ChristensenDalsgaard et al. (1996), which use respectively the OPAL (Rogers et al. 1996), and the MHD (Mihalas et al. 1988), equations of state. The MHD equation of state, based on the "chemical" picture of the plasma, takes into account the effect of excited levels of atoms and ions on the properties of plasma and it also considers a lowest-order Coulomb coupling term through the Debye-Hückel approximation. The OPAL equation of state, in contrast, is based on a "physical" description, in which nuclei and electrons (free or bound) are the only fundamental constituents of the thermodynamic ensemble.

The present helioseismic analyses have been carried out by inversion of preliminary helioseismic data (Rhodes et al. 1998), which include high-degree modes $(l \leq 1000)$, obtained in 1996 by the SOI/MDI instrument on board the SOHO satellite. The set is made up of a very large number of data (7480 modes), which makes the computations slow and very demanding in terms of computer memory. For comparison, we have also inverted another set obtained by the same instrument, which includes only modes with $l \leq 100$ (Schou 1998).

\section{Testing the new surface term}

In order to test the capability of the new $F_{\text {surf }}$ to compensate for the frequency uncertainties arising from the errors of the outermost layers, we have reconstructed the differences in the structure and in the equation of state between the two given reference models by helioseismic inversion of the differences between their theoretical frequencies.

Since the models introduced in the previous section do not include any surface uncertainties, so that $F_{\text {surf }}$ would have been zero, it has been necessary to introduce in Model $S_{\text {MHD }}$ a near-surface modification based on a simple description of the effects of turbulent pressure on the frequencies (Rosenthal 1998).

To get a realistic estimate of the capabilities of the inversions, the artificial mode sets considered comprise the same modes and errors as in the observed data set that includes high-degree modes (Rhodes et al. 1998). 

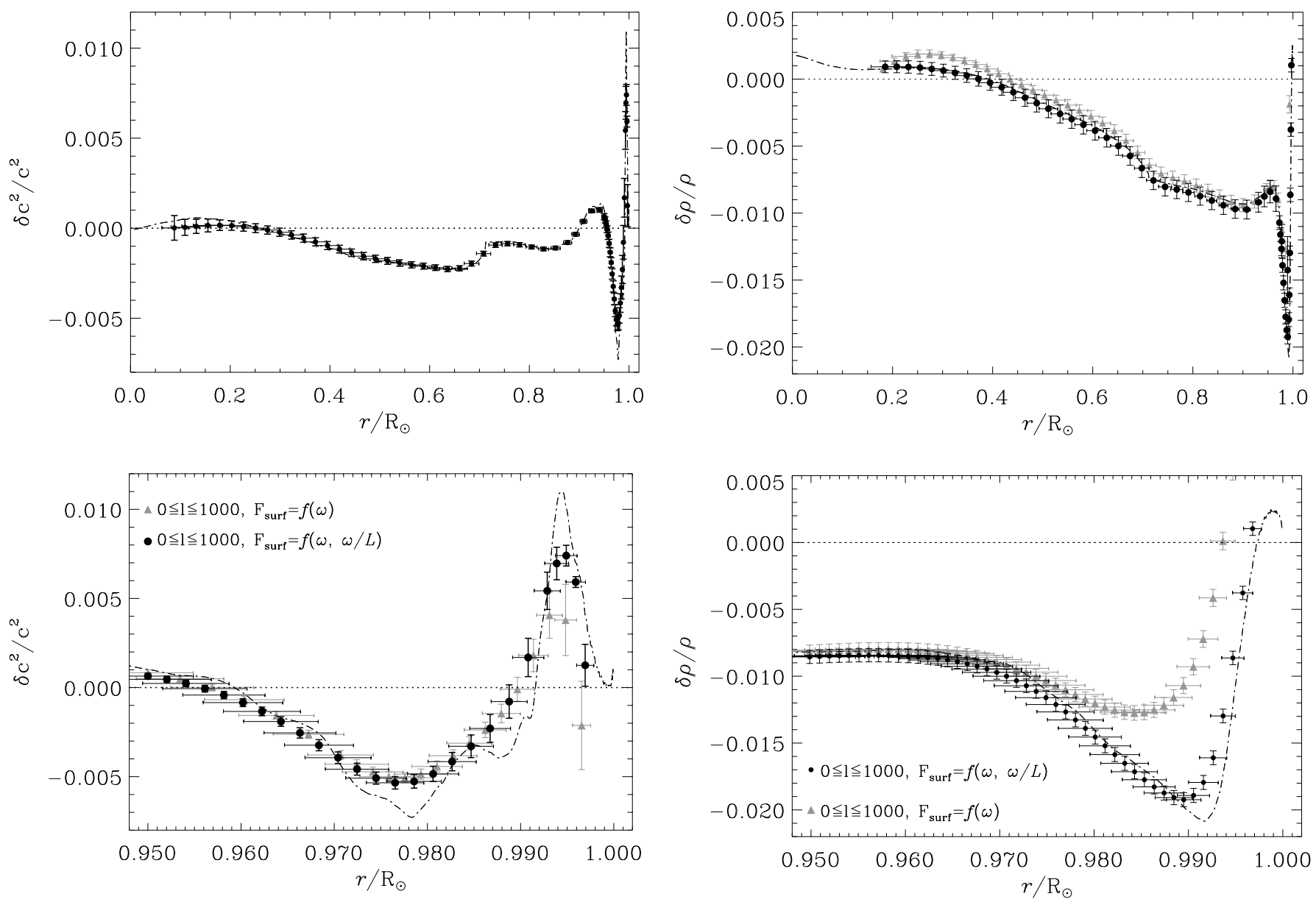

Fig. 3. The relative difference in squared sound speed between models $S_{\mathrm{MHD}}$ and $S_{\mathrm{OPAL}}$, in the sense $\left(S_{\mathrm{MHD}}\right)-\left(S_{\mathrm{OPAL}}\right)$, as obtained by inversion of theoretical frequencies with highdegree modes by considering the new surface term. The vertical errors are $10 \sigma$ propagated errors, while the horizontal bars give a measure of the localization of the averaging kernels. The dotdashed line is the difference in sound speed between the theoretical models used. The detailed behaviour in the sub-surface layers is shown in the lower panel, where the solutions obtained with the use of the new surface term are compared with the results (grey triangles) obtained by using a surface term which depends on frequency alone $F_{\text {surf }}=f(\omega)$.

Figures 3 and 4 show respectively the inferred squared sound-speed and density differences between the two models $S_{\mathrm{MHD}}$ and $S_{\mathrm{OPAL}}$ as functions of the fractional radius. Here the results obtained by adopting the new surface term with $\Lambda=8$ are compared with the solutions obtained by adopting a surface term which is a slowly varying function of frequency $F_{\text {surf }}=f(\omega)$, as is currently used in the inversion of the sets with low- and intermediate-degree modes.

The resulting profiles for the speed of the sound and the density closely reproduce, within the error bars, the exact difference between the given theoretical models. On the contrary, the solutions obtained by adopting a surface

Fig. 4. The relative difference in density between models $S_{\mathrm{MHD}}$ and $S_{\text {OPAL }}$, in the sense $\left(S_{\mathrm{MHD}}\right)-\left(S_{\mathrm{OPAL}}\right)$, as obtained by inversion of theoretical frequencies with high-degree modes by considering the new surface term (Eq. (6)) and by using a surface term which depends on frequency alone $F_{\text {surf }}=f(\omega)$ (grey triangles). The vertical errors are $1 \sigma$ propagated errors, while the horizontal bars give a measure of the localization of the averaging kernels. The dot-dashed line is the difference in density between the theoretical models used. Details in the outer layers are shown in the lower panel.

term which depends on frequency alone are characterized by significant deviations from the actual behaviour in the outer layers and, for density, in the core. We note that the behaviour of the relative squared sound-speed difference obtained by using the new surface term with a Legendre polynomial expansion (Eq. (11)) with $\Lambda=6$ is similar to the solution obtained with $\Lambda=8$.

To illustrate the resolution properties of the inversions, Figs. 5 and 6 show selected averaging kernels. These are clearly extremely well localized, closely reflecting the target kernels $\mathcal{G}\left(r_{0}, r\right)$, while the cross-term kernels are quite small.

Figure 7 shows the true and inferred intrinsic differences in $\Gamma_{1}$ between the equations of state of the 

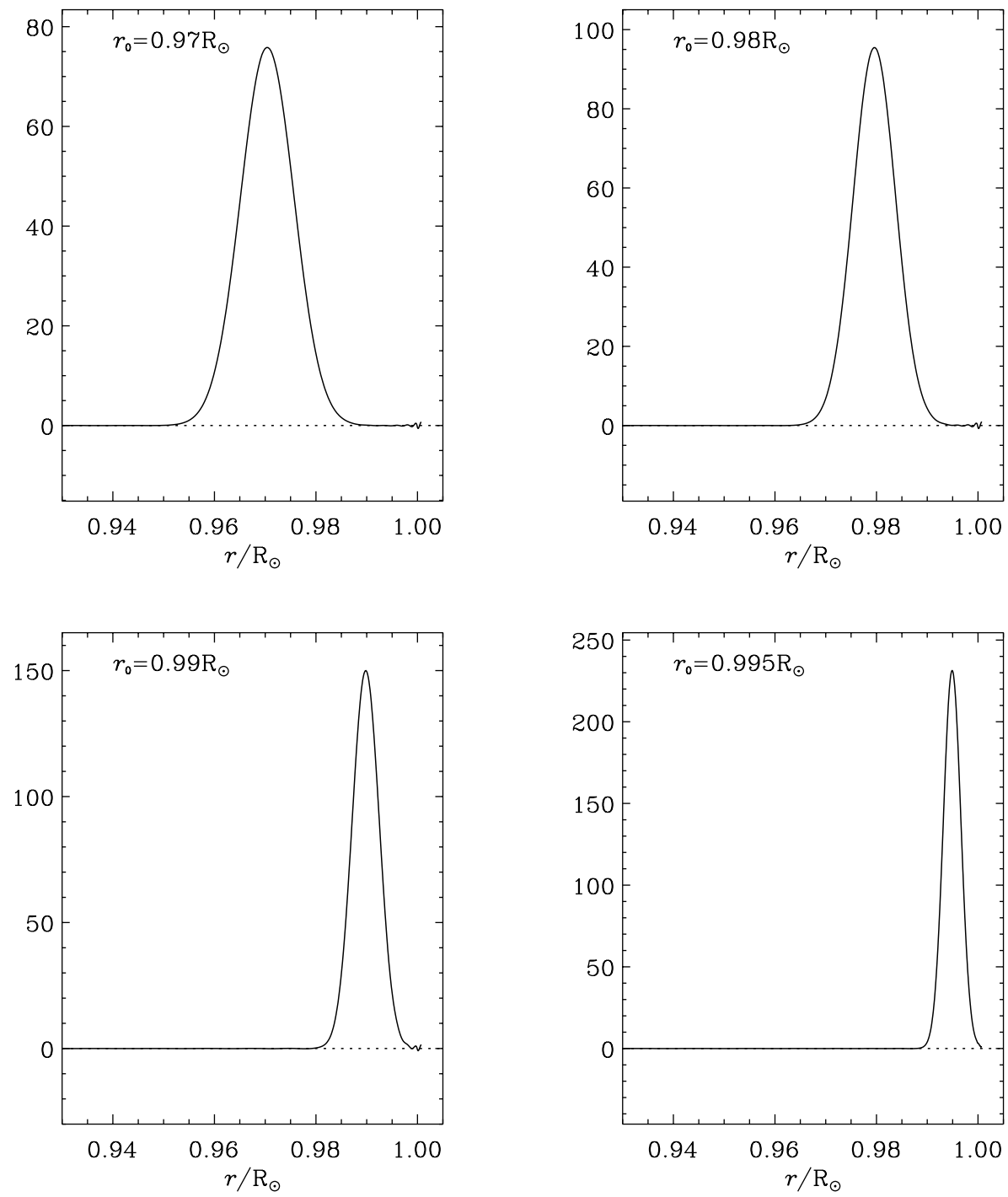

Fig. 5. Averaging kernels for the sound-speed inversion at different target radii (indicated in the panels), as obtained by inverting a set of theoretical frequencies which include high-degree modes and by taking into account the new surface term.

two models. The present mode set, including high-degree modes, allows inversion through the helium ionization zones and parts of the hydrogen ionization zone, clearly reflecting quite accurately the differences arising from the different treatments of the equation of state. Selected averaging kernels, and cross-term kernels $\mathcal{C}_{2}\left(r_{0}, r\right)$ multiplying $\delta Y$, are shown in Fig. 8. The averaging kernels are again quite well localized, even very close to the surface. However, in accordance with Eq. (18), it is not possible entirely to suppress this cross term (in contrast, the first cross-term kernel $\mathcal{C}_{1}\left(r_{0}, r\right)$ is effectively suppressed). In the present model comparison, the difference $\delta Y=6.1 \times 10^{-4}$ is very small and hence the cross term does not significantly affect the inversion.

These tests clearly show the power of high-degree modes and the use of the new surface term to investigate the properties very near the solar surface. Given this success, we now consider differences between the Sun and solar models inferred from analysis of the observed frequencies.

\section{The sound speed and the density for the Sun}

Figures 9 and 10 show the relative squared sound-speed and density differences between the Sun and the model $S_{\text {OPAL }}$ as functions of the fractional radius, obtained by inverting the two sets of observed data. The set of observed frequencies which includes high-degree modes has been inverted with and without the use of the new surface term. In the second case we have adopted again a surface term which is a slowly varying function of frequency $F_{\text {surf }}=f(\omega)$.

As shown in the preceding section, high-degree modes enable inference of properties very near the solar surface, 

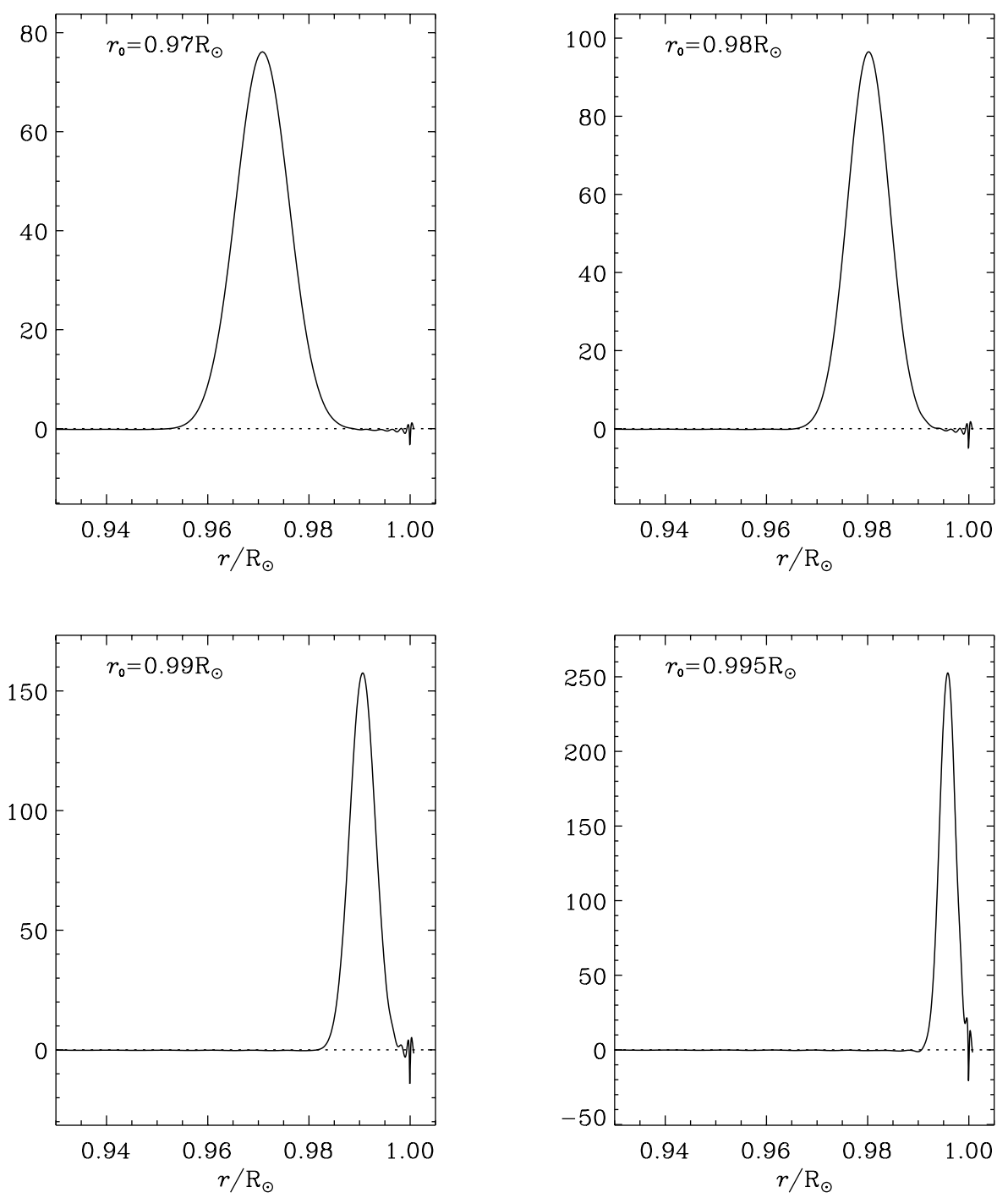

Fig. 6. Averaging kernels for the density inversion at different target radii (indicated in the panels), as obtained by inverting a set of theoretical frequencies which include high-degree modes and by taking into account the new surface term.

through the HeII ionization zone and also part of the HeI ionization zone. In contrast, if only low- and intermediate-degree modes are used, we cannot determine solutions beyond $r \simeq 0.96 R_{\odot}$.

Below the photosphere, for $r \geq 0.95 R_{\odot}$, high-degree modes reveal a large discrepancy between the model and the observed Sun, even considering higher-order asymptotic terms in $F_{\text {surf }}$.

The results for the speed of the sound obtained with the use of the two sets of frequencies are similar in the radiative interior. This indicates that high-degree frequencies have a small influence in the bulk of the Sun when all observed modes of oscillation are considered. It should also be noticed that sound-speed inversion of high-degree modes, using a surface term that depends only on frequency, results in substantial systematic errors in the inversion above $0.97 R_{\odot}$, as was already found in inversion of artificial data (see Sect. 5).

The results for density, plotted in Fig. 10, show that the solution in most of the Sun is relatively little affected by the inclusion of the high-degree modes or the treatment of the surface term. Above $r \simeq 0.5 R_{\odot}$ the use of a surface term depending only on frequency causes a modest change in the results, whereas the solutions with no high-degree modes and with high-degree modes and the full surface term are essentially consistent. In the core, on the other hand, the solutions including high-degree modes show some departure from the inference obtained with just low- and intermediate-degree modes; it should be noticed, however, that use of the new surface term results in a solution that is essentially consistent in the core with the solution obtained with just modes of degree below 100 . 


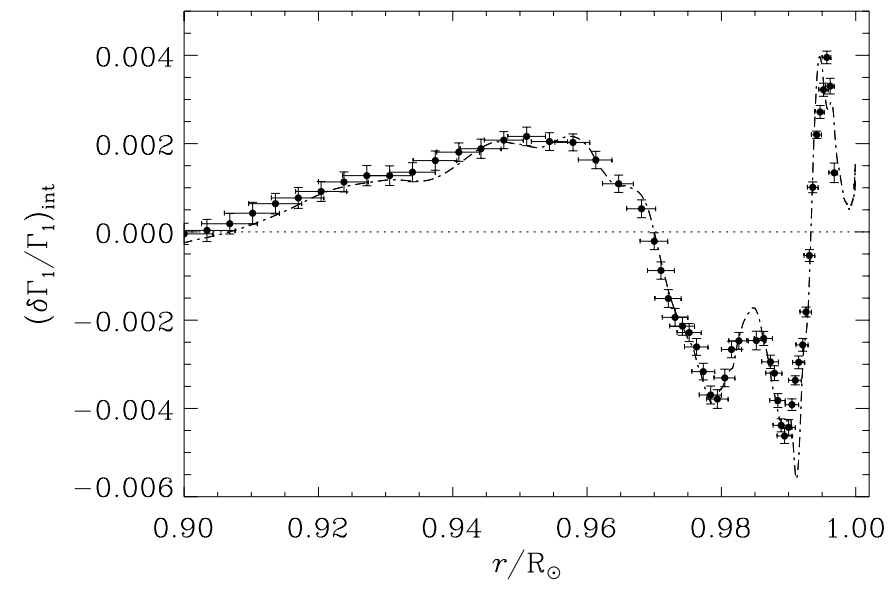

Fig. 7. The relative difference in the adiabatic exponent between models $S_{\mathrm{MHD}}$ and $S_{\mathrm{OPAL}}$, in the sense $\left(S_{\mathrm{MHD}}\right)-$ ( $\left.S_{\text {OpAL }}\right)$, as obtained by inversion of theoretical frequencies with high-degree modes by considering the new surface term presented in Eq. (6). The vertical errors are $2 \sigma$ propagated errors, while the horizontal bars give a measure of the localization of the averaging kernels. The dot-dashed line is the difference in the adiabatic exponent between the theoretical models used.

\section{The equation of state and the helium abundance}

Figure 11 shows the intrinsic differences in $\Gamma_{1}$ between the Sun and the two equations of state considered (OPAL and MHD), as obtained by inversion of the data.

The precise high-degree modes allow determination of variations very near the solar surface, through the He II and also part of the He I ionization zones, while by using only low and intermediate-degree modes, as shown by Basu \& Christensen-Dalsgaard (1997) and by Di Mauro \& Christensen-Dalsgaard (2001), it is impossible to judge the significance of the differences between the two equations of state in the outer layers.

From Fig. 11 we can confirm that, as noticed by Basu et al. (1999), the OPAL equation of state is able to describe better the plasma conditions in the interior of the Sun below $0.97 R_{\odot}$. In the outer layers, the use of high-degree modes provides detailed information, even more strict than the earlier results by Basu et al. (1999) obtained by inverting a set of data with no highdegree modes. We find evidence that the MHD model gives a more accurate description than does the OPAL model in the layers with $0.97 R_{\odot} \leq r \leq 0.99 R_{\odot}$, while above $0.99 R_{\odot}$ the differences become very small and the two equations of state can be considered comparable.

Equation (13) has also been used to determine $\delta Y$, by inverting the data including high-degree modes. The determination of the solar helium abundance is sensitive to the equation of state employed in the reference model, since it is not possible to suppress totally the influence of $\left(\delta \Gamma_{1} / \Gamma_{1}\right)_{\text {int }}$ on $\delta Y$. By using the MHD equation of state, we obtain a value of the helioseismic helium abundance in the convection zone

$Y_{\mathrm{e}}=0.2457 \pm 0.0005$

consistent within errors with the earlier results by Basu \& Antia (1995), by Basu (1998) and by Richard et al. (1998); we note, however, that Kosovichev (1996) found a rather smaller value when using the MHD equation of state. By considering the OPAL equation of state we obtain a value of

$Y_{\mathrm{e}}=0.2539 \pm 0.0005$

in quite good agreement with earlier findings by Basu \& Antia (1995) and by Kosovichev (1996), and just slightly higher (about $2 \%$ ) than the values given by Richard et al. (1998) and by Basu (1998).

\section{Conclusions}

The treatment of the effects of near-surface errors in the inversions is a serious concern. Although a surface term depending just on frequency is adequate for inversions of modes of low and intermediate degree, degree-dependent corrections are required for high-degree modes. The expression for the surface term introduced here (Eq. (6)) may not be completely valid; also, our implementation, in the constraints defined in Eq. (11), may be somewhat problematic. However, it certainly improves the resolution of the inversions by contributing to suppress the errors introduced in computing the theoretical frequencies at the highest degree. By including these modes precise and well-resolved inferences in the highly interesting regions of partial ionization of helium and hydrogen become possible, of substantial importance to tests of the equation of state and determinations of the solar envelope abundances. However, it is important to note that inversions for the outermost layers of the Sun are potentially affected by problems with the determination of sufficiently accurate observed frequencies, free of systematic errors. In fact, the determination of observational frequencies for high-degree modes still suffers from substantial difficulties, related to the merging of power into ridges and the proper treatment of the leakage matrix (e.g. Schou 1998; Rabello-Soares et al. 2001), which may cause systematic errors in the frequencies. Such difficulties could, for example, account for the not insignificant scatter that is still found in the inferred values of the envelope helium abundance, even for determinations utilizing the same equation of state.

Additional problems may be related to the physics of the modes. The expression (5) for the frequency differences assumes that they are caused by differences in the hydrostatic structure between the Sun and the model. In fact, other aspects of the oscillations may affect their frequencies in ways that are not captured by this equation, such as nonadiabaticity, effects of mode excitation or flows. Evidence for such effects is visible in Fig. 1 in 

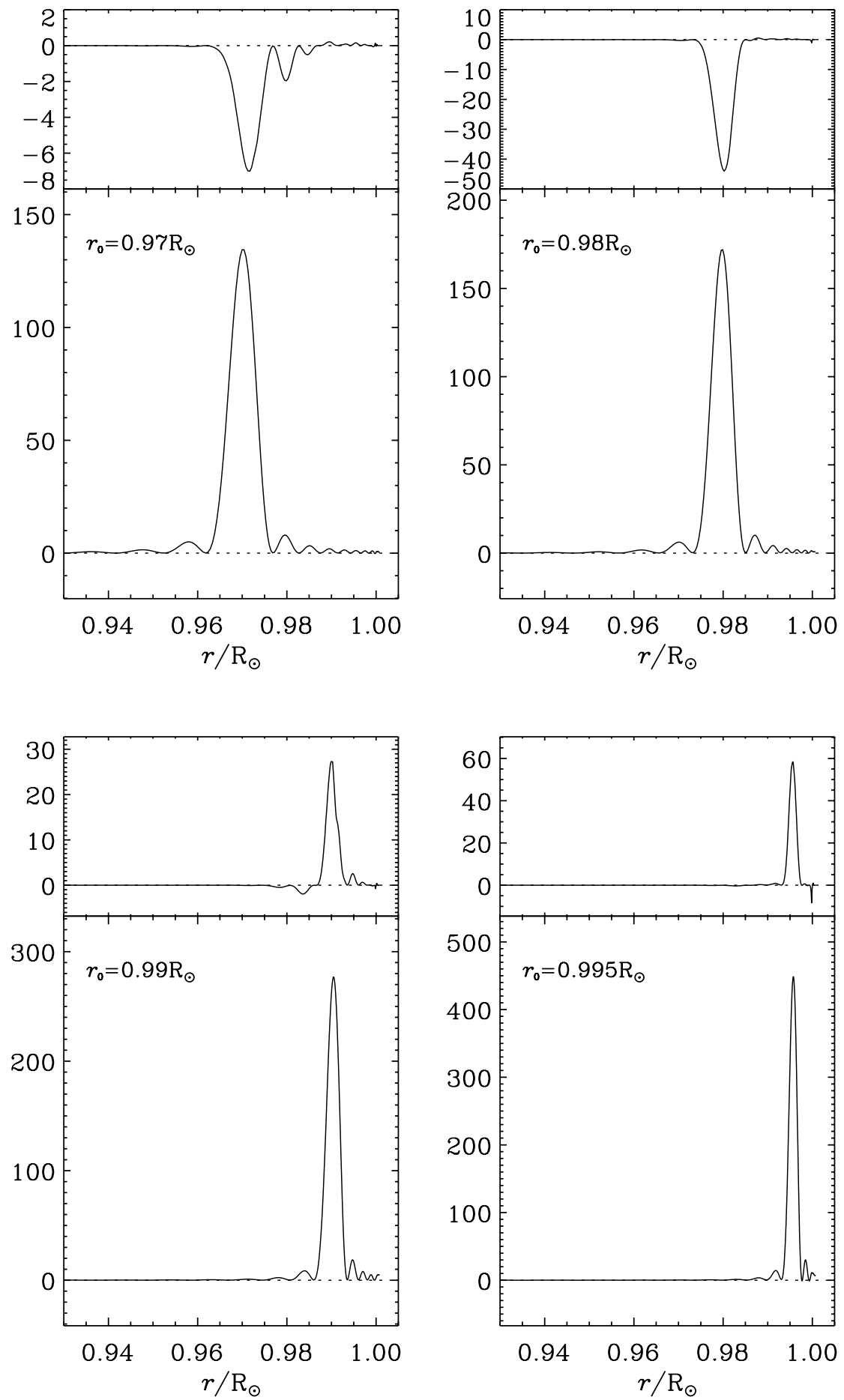

Fig. 8. The lower and upper parts of the panels show respectively averaging kernels and the second cross-term kernel for inversion for $\left(\delta \Gamma_{1} / \Gamma_{1}\right)_{\text {int }}$ as obtained by using high-degree modes and the new surface term (Eq. (6)).

the frequency shift of the f-modes which are unlikely to be caused by changes in the hydrostatic structure (Gough 1993; Chitre et al. 1998). It was shown by Murawski et al. (1998) and Mẹdrek et al. (1999) that this behaviour could be explained by interaction between the modes and turbulent motion in the solar convection zone. Such effect will undoubtedly also influence the acoustic modes and hence must be taken into account before fully reliable inversions can be carried out on the basis of high-degree modes.

Acknowledgements. The work presented here was supported by the Danish National Research Foundation through its establishment of the Theoretical Astrophysics Center. Sarbani Basu is partially supported by NASA Grant \# NAG5-10912. 

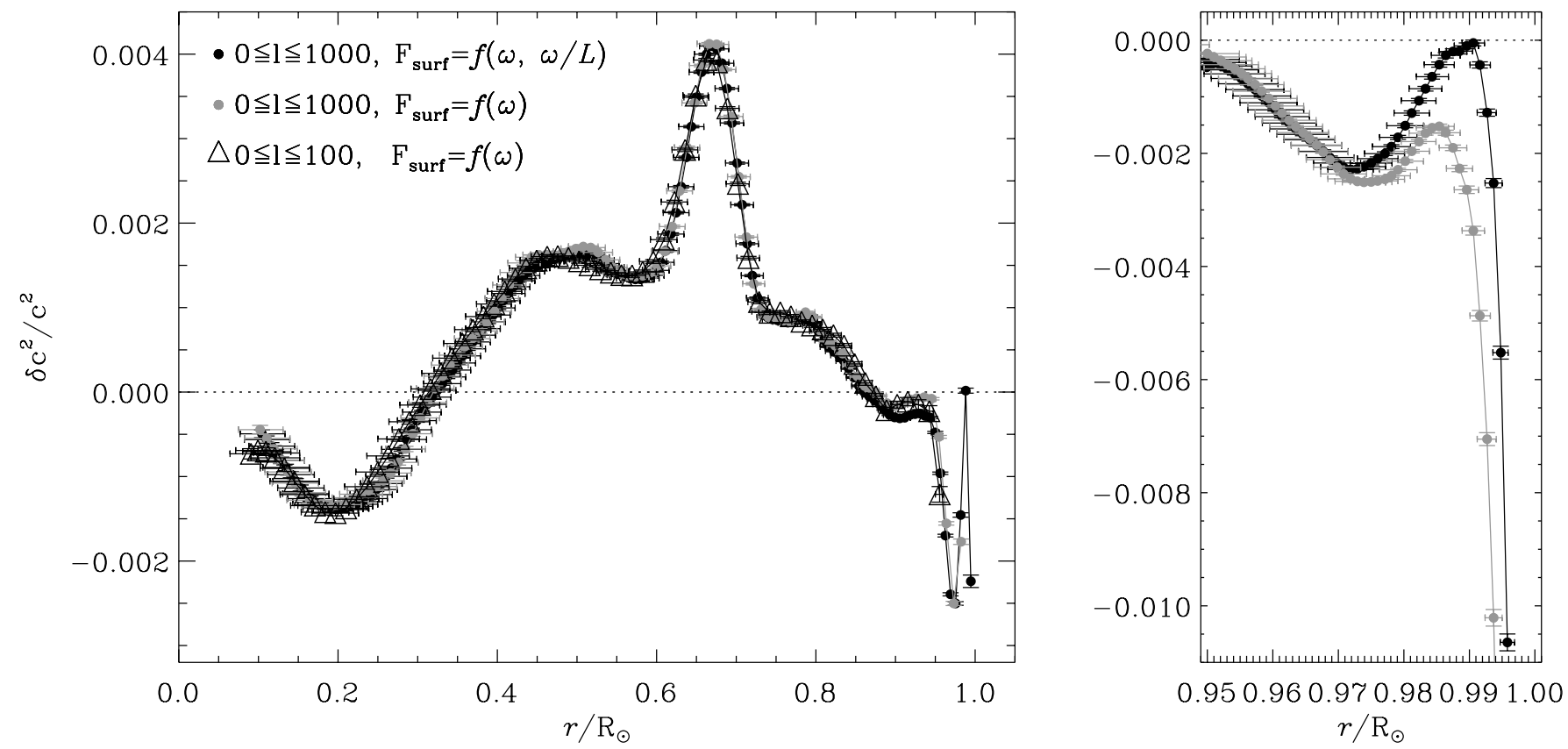

Fig. 9. The relative squared sound-speed difference between the Sun and the standard solar model $S_{\text {OpAL }}$ (Christensen-Dalsgaard et al. 1996), in the sense (Sun) - (model), as obtained by inversion of two different sets of data with high-degree modes (Rhodes et al. 1998) and with no high-degree modes (Schou 1998). The solutions are obtained by considering the new surface term $F_{\text {surf }}=f(\omega, \omega / L)$ as in Eq. (6) with $\Lambda=8$ and a surface term which depends on frequency alone $F_{\text {surf }}=f(\omega)$. The vertical error bars correspond to the standard deviations based on the errors in the mode sets, whereas the horizontal bars give a measure of the localization of the solution. The detailed behaviour in the sub-surface layers is shown in the panel on the right.

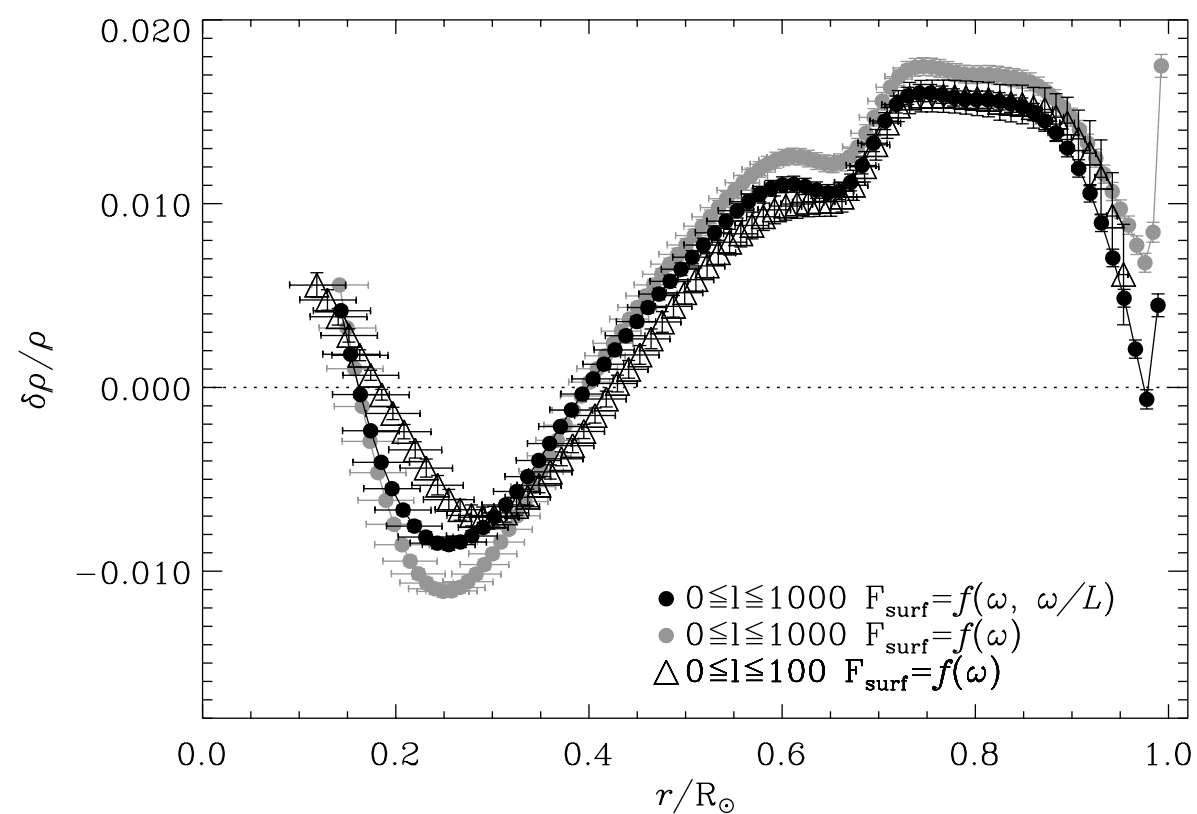

Fig. 10. The relative density differences between the Sun and the standard solar model $S_{\text {OpAL }}$ (Christensen-Dalsgaard et al. 1996), in the sense (Sun) - (model), inferred by using data with high-degree modes (Rhodes et al. 1998) and with no high-degree modes (Schou 1998). The solutions are obtained by considering the new surface term as in Eq. (6) with $\Lambda=8$ and a surface term which depends on frequency alone $F_{\text {surf }}=f(\omega)$. The vertical error bars correspond to the standard deviations based on the errors in the mode sets, whereas the horizontal bars give a measure of the localization of the solution.

\section{References}

Antia, H. M. 1995, MNRAS, 274, 499

Backus, G., \& Gilbert, F. 1968, Geophys. J. RAS, 498, 349

Basu, S. 1998, MNRAS, 298, 719
Basu, S., \& Antia, H. M. 1995, MNRAS, 276, 1402

Basu, S., \& Christensen-Dalsgaard, J. 1997, A\&A, 322, L5

Basu, S., Däppen, W., \& Nayfonov, A. 1999, ApJ, 518, 895

Brodsky, M. A., \& Vorontsov, S. V. 1993, ApJ, 409, 455 


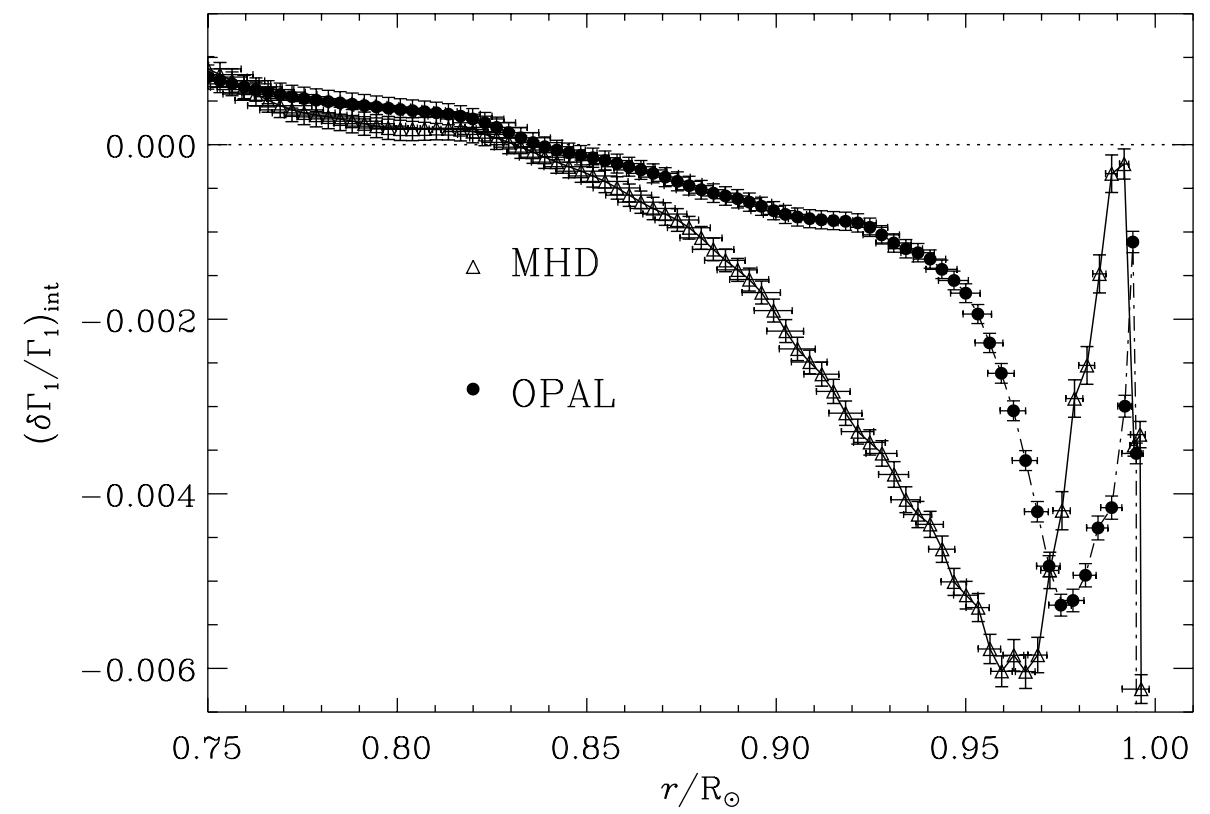

Fig. 11. The intrinsic difference in the adiabatic exponent $\Gamma_{1}$ between the Sun and the OPAL (Rogers et al. 1996) equation of state (filled circle) and the Sun and the MHD (Mihalas et al. 1988) equation of state (open triangles), in the sense (Sun) (model), obtained by inversion of a set of data by Rhodes et al. (1998), which includes high-degree modes, and by considering the new surface term as presented in Eq. (6). The vertical errors are $2 \sigma$ propagated errors, while the horizontal bars give a measure of the localization of the averaging kernels.

Chitre, S. M., Christensen-Dalsgaard, J., \& Thompson, M. J. 1998 , in Structure and dynamics of the interior of the Sun and Sun-like stars, Proc. SOHO 6/GONG 98 Workshop, ed. S. G. Korzennik, \& A. Wilson, ESA SP-418 (ESA Publications Division, Noordwijk, The Netherlands), 141

Christensen-Dalsgaard, J., \& Berthomieu, G. 1991, in Solar interior and atmosphere, ed. A. N. Cox, W. C. Livingston, \& M. Matthews, Space Science Series (University of Arizona Press), 401

Christensen-Dalsgaard, J., \& Thompson, M. J. 1997, MNRAS, 284,527

Christensen-Dalsgaard, J., Däppen, W., Ajukov, S. V., et al. 1996, Science, 272, 1286

Christensen-Dalsgaard, J., Gough, D., \& Thompson, M. 1989, MNRAS, 238, 481

Di Mauro, M. P., \& Christensen-Dalsgaard, J. 2001, in IAU Symp. 203, Recent Insights into the Physics of the Sun and Heliosphere: Highlights from SOHO and Other Space Missions, ed. P. Brekke, B. Fleck, \& J. B. Gurman (ASP, San Francisco), 177

Dziembowski, W. A., Pamyatnykh, A. A., \& Sienkiewicz, R. 1990, MNRAS, 244, 542

Dziembowski, W. A., Pamyatnykh, A. A., \& Sienkiewicz, R. 1992, Acta Astron., 42, 5

Gough, D. O. 1984, in Conference on Oscillations as a Probe of the Sun's Interior, Mem. Soc. Astron. Ital., 55, 13

Gough, D. O. 1993, in Astrophysical fluid dynamics, Les Houches Session XLVII, ed. J.-P. Zahn, \& J. Zinn-Justin (Elsevier, Amsterdam), 399

Gough, D. O., \& Vorontsov, S. V. 1995, MNRAS, 273, 573

Kosovichev, A. G. 1996, Bull. Astron. Soc. India, 24, 355

Kosovichev, A. G., Christensen-Dalsgaard, J., Däppen, W., et al. 1992, MNRAS, 259, 536

Mędrek, M., Murawski, K., \& Roberts, B. 1999, A\&A, 349, 312
Mihalas, D., Däppen, W., \& Hummer, D. G. 1988, ApJ, 456, 902

Murawski, K., Duvall, T. L., \& Kosovichev, A. G. 1998, in Structure and dynamics of the interior of the Sun and Sunlike stars; Proc. SOHO 6/GONG 98 Workshop, ed. S. G. Korzennik, \& A. Wilson, ESA SP-418 (ESA Publications Division, Noordwijk, The Netherlands), 825

Pijpers, F. P., \& Thompson, M. J. 1992, A\&A, 262, L33

Pijpers, F. P., \& Thompson, M. J. 1994, A\&A, 281, 231

Rabello-Soares, M. C., Basu, S., \& Christensen-Dalsgaard, J. 1999, MNRAS, 309, 35

Rabello-Soares, M. C., Christensen-Dalsgaard, J., Basu, S., \& Di Mauro, M. P. 2000, Sol. Phys., 193, 345

Rabello-Soares, M. C., Korzennik, S. G., \& Schou, J. 2001, in Helio- and Asteroseismology at the Dawn of the Millennium, Proc. SOHO 10 / GONG 2000 Workshop, ed. A. Wilson, ESA SP-464 (ESA Publications Division, Noordwijk, The Netherlands), 129

Richard, O., Dziembowski, W. A., Sienkiewicz, R., \& Goode, P. R. 1998, A\&A, 338, 756

Rhodes, E. J., Reiter, J., Kosovichev, A. G., Schou, J., \& Scherrer, P. H. 1998, in Structure and Dynamics of the Interior of the Sun and Sun-like Stars, ed. S. G. Korzennik, \& A. Wilson, ESA SP-418 (ESA Publications Division, Noordwijk, The Netherlands), 73

Rogers, F. J., Swenson, F. J., \& Iglesias, C. A. 1996, ApJ, 331, 815

Rosenthal, C. 1998, in IAU Symp. 181, Sounding solar and stellar interiors, Poster volume, ed. J. Provost, \& F.-X. Schmider (Université de Nice), 54

Schou, J. 1998, in Structure and Dynamics of the Interior of the Sun and Sun-like Stars, ed. S. G. Korzennik, \& A. Wilson, ESA SP-418 (ESA Publications Division, Noordwijk, The Netherlands), 47 